\title{
Robust gain-scheduled blending control of raw-mix quality in cement industries
}

\author{
Vinicius de Oliveira ${ }^{1}$, Michael Amrhein ${ }^{2}$ and Alireza Karimi ${ }^{1}$
}

\begin{abstract}
This paper addresses the control of the blending process in cement industries. This process can be modeled by a nonlinear multivariable system with large parametric uncertainty. Using a specific transformation, a linear parameter varying (LPV) model with set-points as scheduling parameters is developed. Moreover, the model uncertainty originated from the stochastic variation of the composition of the input materials is represented as a polytopic multimodel uncertainty. Then a multivariable gain-scheduled robust controller is designed by convex optimization to control the quality of the raw mix in the blending process. The control performance is illustrated by simulation and compared with a robust controller based on a nominal model.
\end{abstract}

\section{INTRODUCTION}

The production of cement consists of many processing steps such as crushing, input material blending and grinding of the input materials, chemical conversion to clinker, and grinding of the clinker to the final cement. The focus of this work is the input material blending process whose task is to mix the input materials in order to produce raw mix which is ground in the raw mill to produce raw meal for the kiln. It is well known that the oxide compositions of the raw meal influence significantly the quality of the clinker and the kiln operation. The goal of a blending controller is to continuously maintain the raw mix quality on the desired set-points by adjusting the weigh feeder proportions of the input materials. Nonetheless, the control of the quality of the raw mix is not a trivial task due to stochastic variation of the chemical composition of the input materials, e.g., if they are mined from different queries. The composition variations may lead to undesired behaviour of the control system such as off-sets and oscillations. Moreover, it is shown that the system parameters also vary with changes of the operating points. Therefore, the system can be described by a linear parameter varying (LPV) model with parametric uncertainty.

Various approaches of blending control of the raw mix are available in the literature. In [1], a model predictive control (MPC) is proposed based on an identified parametric multivariable model. An adaptive control of the raw mill system is described in [2], where the composition of the input materials are estimated on-line and used in a decoupling network. The singular value decomposition algorithm (SVD) to compute the feeder proportions of the raw mix was introduced in [3]. A self-tuning MIMO composition control strategy in which a multivariable model is estimated on-line and used to update the controller parameters is investigated

\footnotetext{
${ }^{1}$ Automatic Control Laboratory at Ecole Polytechnique Fédérale de Lausanne (EPFL), Switzerland. ${ }^{2}$ Online Control Ltd. Lausanne, Switzerland. Corresponding author: Alireza Karimi: alireza.karimi@epfl.ch
}

by [4]. Gradient-based predictive controller and non-linear optimization techniques are used to compute the proportions of the input materials in [5]. An optimal MIMO controller with input saturation using linear programming is presented in [6].

In this paper, an approach addressing the decoupling, the parametric uncertainty and the dependence of the dynamics on the operating point will be proposed. A robust gainscheduled MIMO blending controller will be illustrated for three input material feeders using on-line measurements. The controller will be robust with respect to the variation in the composition of the input materials and its gains will be function of the set-points of the quality modules of the raw mix. The controller design method used is based on loopshaping in the Nyquist diagram under infinity norm constraints on closed-loop transfer functions and was proposed in [7], [8]. The decoupling controllers and the controller for the decoupled controlled system are designed simultaneously. In this paper an extension of this design method is presented which handles uncertain LPV models by using robust gainscheduled MIMO controllers with decoupling elements.

The article is organized as follows: the process and the control problem are described in Section II; Section III studies the robust control design method and the proposed extension for LPV systems; Section IV presents a case study where the proposed robust controller method is used to solve the feeder blending control problem.

\section{PROCESS DESCRIPTION}

The plant considered in this work consists of input materials, such as limestone, marl, iron corrective, etc. that are individually supplied to the plant by different weigh feeders. Each of the input materials has a specific composition of calcium oxide $(\mathrm{CaO})$, silicon dioxide $\left(\mathrm{SiO}_{2}\right)$, aluminum oxide $\left(\mathrm{Al}_{2} \mathrm{O}_{3}\right)$ and iron oxide $\left(\mathrm{Fe}_{2} \mathrm{O}_{3}\right)$. The flow-rate of each weigh feeder is controlled by a dosage controller based on a scale and the set-point adjusted by the blending controller. An on-line analyzer (OLA; e.g., prompt gamma neutron activation - PGNA) measures on-line the composition of the raw mix.

The focus of this work is the design of blending controllers of the raw mix quality. Fig. 1 depicts the schematic of the blending process. The goal of the blending controller is to adjust the proportions of the input materials (the manipulated inputs) such that the raw mix quality modules (the controlled outputs), a set of nonlinear functions of the raw mix composition, are on the desired set-points. 


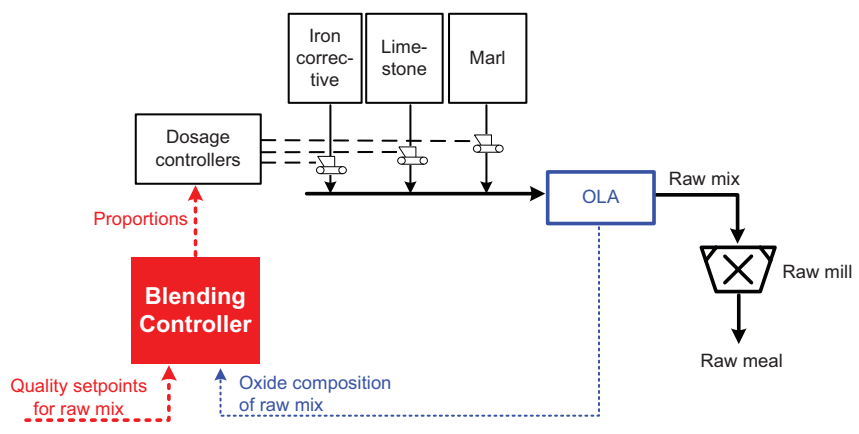

Fig. 1. Blending control problem

There are some difficulties in this process that make it a challenging control problem. First, the static gains of the system depend on the chemical compositions of the input materials, which are uncertain and may vary considerably. Second, the system is multivariable with strong coupling between the outputs. Third, the system has LPV behaviour since the static gains of the system are also functions of the set-points. Fourth, the quality modules are nonlinear functions of the feeder proportions. Finally, the manipulated inputs are constrained due to physical, chemical or economical restrictions. The challenges are summarized in the following.

1) Uncertainty of the input material compositions

2) Strongly coupled plant

3) LPV behaviour due to operating points

4) Nonlinear behaviour

5) Constrained inputs

The first three problems will be handled by a robust MIMO gain-scheduled control design method that will be presented in the next section. The nonlinearities will be dealt with by using a transformation proposed in [6] which transforms the nonlinear quality modules into an affine form when the set-points are given. The constraints of the input will be iteratively checked by simulations since introducing these constraints in the control design technique would only produce more conservative controller.

\section{CONTROL DESIGN METHOD}

In this section, we will first present the method given in [7] to design fixed-order MIMO controllers. Then, an extension of the method will be proposed to design robust gain-scheduled controllers.

As it has been discussed, the blending process can be described as a MIMO system. One approach that can be used to design controllers for MIMO systems consists of a two-step technique: in the first step, the MIMO system is transformed into a diagonally dominant system using a decoupler. Once the system is diagonally dominant, the techniques used for SISO systems are used to design decoupled controllers for each diagonal element of the MIMO system.

Galdos et al. [7] proposed a method to design robust MIMO controllers for spectral models using convex optimization. One of the main features of this technique is that a one-step convex optimization algorithm can be used to simultaneously design decoupling controller and the decoupled controlled system.

\section{A. Design of robust MIMO controllers}

Consider the class of fixed-order linearly parametrized MIMO controllers and the systems described by nonparametric spectral models. If a parametric model is available a similar technique can be applied. The class of continuoustime or discrete-time LTI-MIMO systems with bounded infinity norm is given by

$$
\mathbb{G}:=\left\{G_{i}\left(j \omega_{k}\right) \quad i=1, \ldots, m ; \quad k=1, \ldots, N\right\}
$$

where $m$ is the number of models in the set and $G_{i}\left(j \omega_{k}\right)$ is a $n_{0} \times n_{i}$ matrix of complex values with $n_{i}$ the number of inputs and $n_{0}$ the number of outputs. $N$ should be large enough so that it will give a good approximation of the open-loop frequency response of the system in the Nyquist diagram. For simplicity, consider only the nominal model $G$ which belongs to the class.

Consider the class of multivariable controllers given by a $n_{i} \times n_{0}$ matrix $K(s)$ whose elements $K_{p q}(s)$ for $p=$ $1, \ldots, n_{i}$ and $q=1, \ldots, n_{0}$ are linearly parametrized as follows:

$$
K_{p q}(s)=\rho_{p q}^{T} \phi_{p q}(s)
$$

where

$$
\begin{gathered}
\rho_{p q}^{T}=\left[\rho_{p q 1}, \rho_{p q 2}, \ldots, \rho_{p q n}\right] \\
\phi_{p q}^{T}=\left[\phi_{p q 1}(s), \phi_{p q 2}(s), \ldots, \phi_{p q}(s)\right]
\end{gathered}
$$

and $\rho_{p q}^{T}$ corresponds to the controller parameters and $\phi_{p q}^{T}$ is a vector containing the basis functions. For example, using this parametrization a PID controller could be described by

$$
\rho^{T}=\left[K_{p}, K_{i}, K_{d}\right] \quad, \quad \phi^{T}=\left[1, \frac{1}{s}, \frac{s}{T_{d} s+1}\right]^{T}
$$

The Generalized Nyquist Stability criterion states that the feedback system will be stable if and only if the net sum of the anticlockwise encirclements of the critical point $(-1+j 0)$ by the set of eigenvalues of the $n_{0} \times n_{0}$ open-loop transfer matrix $L(j \omega, \rho)=G(j \omega) K(j \omega)$ is equal to the number of unstable poles of $L(j \omega)$. The eigenvalues of the matrix $L\left(j \omega_{k}, \rho\right)$ at each frequency is a non-convex function of the controller parameters $\rho$. Reminding that every eigenvalue lies within at least one Gershgorin band [9], sufficient conditions of the closed-loop stability can be obtained and written as a set of convex constraints. Given a spectral model $G(j \omega)$, it can be proved [7] that the controller $K(s)$ stabilizes the closed-loop if

$$
\begin{gathered}
r_{q}\left(\omega_{k}, \rho\right)-\frac{R_{e}\left\{\left[1+L_{D q}\left(-j \omega_{k}\right)\right]\left[1+L_{q q}\left(j \omega_{k}, \rho\right)\right]\right\}}{\left|\left[1+L_{D q}\left(j \omega_{k}\right)\right]\right|}<0 \\
q=1, \ldots, n_{0} \quad k=1, \ldots, N \quad \text { (5) }
\end{gathered}
$$

where $L_{D}(j \omega)$ is a diagonal desired open-loop transfer matrix chosen such that the number of anticlockwise encirclements of the critical point on the Nyquist plot of the set 
of eigenvalues is equal to the number of unstable poles of $G(j \omega)$. The $q$-th Gershgorin band $r_{q}$ of $L(j \omega)$ is defined as

$$
r_{q}\left(\omega_{k}, \rho\right)=\sum_{p=1, p \neq q}^{n_{0}}\left|L_{p q}\left(j \omega_{k}, \rho\right)\right|
$$

The stability condition given by Eq. (5) adds some conservatism since the eigenvalues are no longer located in a point but are allowed to be in a circle of radius $r_{q}\left(\omega_{k}, \rho\right)$. To reduce the conservatism, one can minimize the radius $r_{q}\left(\omega_{k}, \rho\right)$ which is equivalent to minimize the off-diagonal components of the open-loop transfer function matrix $L(j \omega, \rho)$. Thus, it is judicious to minimize the following criterion

$$
J(\rho)=\left\|L(\rho)-L_{D}\right\|_{2}^{2}
$$

This way the off-diagonal elements will be minimized which helps to decouple the system. Approximating the cost function $J(\rho)=\left\|L(\rho)-L_{D}\right\|_{2}^{2}$ by $\sum_{\omega}\left\|L(j \omega, \rho)-L_{D}(j \omega)\right\|_{F}$ where $\|\cdot\|_{F}$ is the Frobenius norm and considering the whole set of models $\mathbb{G}$, it leads to the following convex optimization problem

$$
\min _{\rho} \sum_{i=1}^{m} \sum_{k=1}^{N}\left\|L_{i}\left(j \omega_{k}, \rho\right)-L_{D i}\left(j \omega_{k}\right)\right\|_{F}
$$

subject to

$$
\begin{gathered}
r_{q}\left(\omega_{k}, \rho\right)-\frac{R_{e}\left\{\left[1+L_{D q i}\left(-j \omega_{k}\right)\right]\left[1+L_{q q}\left(j \omega_{k}, \rho\right)\right]\right\}}{\left|\left[1+L_{D q i}\left(j \omega_{k}\right)\right]\right|}<0 \\
q=1, \ldots, n_{0} \quad k=1, \ldots, N \quad i=1, \ldots, m
\end{gathered}
$$

\section{B. Extension to design MIMO LPV controllers}

A natural extension of this method is to consider a gainscheduling controller in which the controller's gains are function of a scheduling vector parameter $\theta$ [10]. Consider the multivariable controller $K(s, \theta)$ whose elements $K_{p q}(s, \theta)$ are linearly parametrized by

$$
K_{p q}(s, \theta)=\rho_{p q}^{T}(\theta) \phi_{p q}(s)
$$

where the basis function vector $\phi_{p q}$ is defined on Eq. (4) and $\rho_{p q}^{T}(\theta)$ is given by

$$
\rho_{p q}^{T}(\theta)=\left[\rho_{p q 1}(\theta), \rho_{p q 2}(\theta), \ldots, \rho_{p q n}(\theta)\right]
$$

Every gain is a polynomial function of order $\delta$ of the scheduling parameter and is defined as

$$
\rho_{p q i}(\theta)=\left(\nu_{p q i, \delta}\right)^{T} \theta^{\delta}+\ldots+\left(\nu_{p q i, 1}\right)^{T} \theta+\nu_{p q i, 0}
$$

where $\theta^{k}$ denotes element-by-element power of $k$ of vector $\theta$.

If an LPV model is available, there will be an infinity number of models corresponding to different values of the scheduling parameters. This problem can be solved by gridding $\theta$ leading to $m_{g}$ different LTI models. In the case of multimodel uncertainty, the procedure should be repeated for each one of the $m_{u}$ uncertain LPV models. The design procedure is stated in the following.
Let $L(s, \theta)=G(s, \theta) K(s, \theta)$ be the open loop transfer function. The gain-scheduled controller is thus obtained from the following convex optimization problem:

$$
\min _{\rho} \sum_{i=1}^{m_{u}} \sum_{l=1}^{m_{g}} \sum_{k=1}^{N}\left\|L_{i}\left(j \omega_{k}, \theta_{l}\right)-L_{D i}\left(j \omega_{k}\right)\right\|_{F}
$$

subject to

$$
\begin{aligned}
& \quad r_{q i}\left(\omega_{k}, \theta_{l}\right)-\frac{R_{e}\left\{\left[1+L_{D i q}\left(-j \omega_{k}\right)\right]\left[1+L_{i q q}\left(j \omega_{k}, \theta_{l}\right)\right]\right\}}{\left|\left[1+L_{D i q}\left(j \omega_{k}\right)\right]\right|}<0 \\
& q=1, \ldots, n_{0} \quad k=1, \ldots, N, \quad l=1, \ldots, m_{g}, i=1, \ldots, m_{u}
\end{aligned}
$$

where $\theta_{l}$ is a vector with particular values of the scheduling parameters $\theta$ obtained from the gridding. The proposed procedure will be illustrated in the next section by the threefeeder blending problem.

\section{CASE STUDY}

First, the model of the process is presented. Next, the modeling of the uncertainty in the composition of the input material is illustrated. Finally, the robust gain-scheduled controller is designed and evaluated for different conditions.

\section{A. Process Model}

Consider a blending process comprising three input materials namely, limestone, marl and iron corrective that are fed by three weigh feeders. The oxide composition of the raw mix is measured by an OLA whose sampling time is $T_{s}=2$ $\mathrm{min}$. The goal of the controller is to adjust the proportions of limestone, marl and iron corrective, respectively, $z_{1}, z_{2}$ and $z_{3}$. The quality of the raw mix is related to the value of the following quality modules, namely, limestone saturation $\alpha$ (LS) and aluminum ratio $\gamma(\mathrm{AR})$ or iron module (IR) which are defined as

$$
\begin{aligned}
& \alpha=\alpha\left(x_{1}, x_{2}, x_{3}, x_{4}\right)=\frac{100 x_{1}}{2.8 x_{2}+1.18 x_{3}+0.65 x_{4}} \\
& \gamma=\gamma\left(x_{3}, x_{4}\right)=\frac{x_{3}}{x_{4}}
\end{aligned}
$$

where $x_{1}, x_{2}, x_{3}, x_{4}$ respectively stand for the weight fractions of $\mathrm{CaO}, \mathrm{SiO}_{2}, \mathrm{Al}_{2} \mathrm{O}_{3}$, and $\mathrm{Fe}_{2} \mathrm{O}_{3}$ of the raw mix.

Let $z=\left[z_{1}, z_{2}, z_{3}\right]^{T}$ be the vector of feeder proportions that add up to unity leading to the following constraint:

$$
z_{1}+z_{2}+z_{3}=1
$$

Without loss of generality, the manipulated inputs are chosen to be $z_{1}$ and $z_{2}$ whereas the feeder proportion $z_{3}$ is be computed from Eq. (14).

Defining $r=[\alpha, \gamma]^{T}$ the vector of quality modules to control, the nonlinear input-output steady-state relation is given by $r=f(W z)$, where $W$ is a $4 \times 3$ composition matrix in which each column represents the composition of the corresponding input material as it is shown, for instance, in Table I, $f$ represents the functions given in Eq. (13). Note that $x=W z$ where $x=\left[x_{1}, x_{2}, x_{3}, x_{4}\right]^{T}$.

By making use of the set-points vector $r_{c}=\left[\alpha_{c}, \gamma_{c}\right]$, the quality modules can be transformed into a linear form by applying the transformation matrix $M\left(r_{c}\right)$ to the measured 
compositions vector $x$. The transformed qualities is then $y=$ $M\left(r_{c}\right) x$ where

$$
M\left(r_{c}\right)=\left[\begin{array}{cccc}
100 & -2.8 \alpha_{c} & -1.18 \alpha_{c} & -0.65 \alpha_{c} \\
0 & 0 & 1 & -\gamma_{c}
\end{array}\right]
$$

Note that by using this transformation, the input-output static relation becomes $y=M\left(r_{c}\right) W z$, which is affine in $z$ for a fixed set-point vector $r_{c}$. The set-point vector of the transformed qualities is always $y_{c}=[0,0]^{T}$.

The $2 \times 2$ transfer function matrix $G(s)$ between the feeder proportions $z$ and the transformed qualities $y$ is given by

$$
G(s)=\frac{\Delta y(s)}{\Delta z(s)}=\left[\begin{array}{ll}
k_{11} g_{11}(s) & k_{12} g_{12}(s) \\
k_{21} g_{21}(s) & k_{22} g_{22}(s)
\end{array}\right]
$$

where the unitary-gains transfer functions $g_{i j}$ account for the feeder dynamics, transport and measurement delays, the noise filter dynamics of the $j$ th feeder on the $i$ th transformed quality module. The static gains of the system are given by:

$$
\begin{array}{ll}
k_{11}=\frac{\partial y_{1}}{\partial z_{1}}=a_{11} \alpha_{c}+b_{11} & k_{12}=\frac{\partial y_{1}}{\partial z_{2}}=a_{12} \alpha_{c}+b_{12} \\
k_{21}=\frac{\partial y_{2}}{\partial z_{1}}=a_{21} \gamma_{c}+b_{21} & k_{22}=\frac{\partial y_{2}}{\partial z_{2}}=a_{22} \gamma_{c}+b_{22}
\end{array}
$$

Note that the gains are affine functions of the quality module set-points and the coefficients $a_{i j}$ and $b_{i j}$ depend on the oxide compositions of the input materials. This means that the system is LPV due to variation of the set-points and has parametric uncertainty. The modeling of the uncertainties will be shown in the next section.

\section{B. Modeling the uncertainty}

The variation of the input material compositions, given by matrix $W$, is one of the main issues in blending processes since it affects directly the static gains of the system. A first formulation to design a robust controller considers the variance of each entry of $W$ independently. However, it would lead to conservatism if strong correlation in the variances is observed. Instead, a method applying the well known principal component analysis (PCA) to identify patterns in the varying composition of each input material is proposed.

Consider a composition matrix $W$ given by

$$
W=\left[w_{1}, w_{2}, \ldots, w_{n_{f}}\right]
$$

where the $4 \times 1$ vector $w_{e}$ contains the composition of the eth input material and $n_{f}$ is the number of feeders. Each vector can be written as

$$
w_{e}=w_{e 0}+\Delta w_{e}
$$

where $\Delta w_{e}$ represents the variation around the average composition $w_{e 0}$. The goal is to compute the bounds of the variation $\Delta w_{e}$ by analyzing a set of measurements of the compositions of the input materials using PCA.

Consider the composition $w_{e}$ of the eth input material be analyzed for $L$ samples leading to an $L \times 4$ matrix $\bar{W}_{e}$. The matrix of variations can be defined by

$$
\Delta \bar{W}_{e}=\bar{W}_{e}-\mathbf{1}_{L} w_{e 0}^{T}
$$

where $1_{L}$ is a $L \times 1$ vector of ones and $w_{e 0}$ is the column mean of $\overline{W_{e}}$. Applying PCA leads to an approximation of $\Delta \overline{W_{e}}$ :

$$
\Delta \bar{W}_{e} \simeq T_{a} P_{a}^{T}
$$

where $T_{a}$ is an $L \times a$ matrix of the first $a$ scores and $P_{e 1}^{T}$ the $a \times 4$ matrix of the first $a$ loadings. Here, $a=1$ for all the input materials since the first principal component (PC) of limestone retains already $96.2 \%$ of the variance, that of marl $90.94 \%$, and that of the iron corrective $88.7 \%$. Computing the standard deviation of the scores vector, $\sigma_{e}=\sigma\left(T_{1}\right)$, the lower and upper bounds of the variation $\Delta w_{e}$ can be defined as $\Delta w_{e}^{-} \simeq-2 \sigma_{e} P_{1}^{T}$ and $\Delta w_{e}^{+} \simeq 2 \sigma_{e} P_{1}^{T}$.

The last step is to model the uncertain composition matrix $W$. For this particular case with three input materials, the matrix becomes

$$
W=\left[\left(w_{10}+\Delta w_{1}\right),\left(w_{20}+\Delta w_{2}\right),\left(w_{30}+\Delta w_{3}\right)\right]
$$

Considering all compositions vary within the vertices of the respective intervals

$$
\Delta w_{e} \in\left[\Delta w_{e}^{-}, \Delta w_{e}^{+}\right], \quad e=1, \ldots, 3
$$

then $2^{3}=8$ matrices $W_{i}$ for $i=1, \ldots, 8$ are found. Note that if each composition had been treated independently, $2^{12}=4096$ matrices would have been computed, increasing considerably the computational complexity in the design. The standard deviation of the first scores of the three feeders are respectively $\sigma_{1}=1.28, \sigma_{2}=2.99$ and $\sigma_{3}=1.59$.

Finally, the composition matrices $W_{i}$ are used to generate $8 \mathrm{LPV}$ models in the form $G_{i}(\theta)$, where $\theta$ is the vector of scheduling parameters such that $\theta=\left[\alpha_{c}, \gamma_{c}\right]^{T}$. These models are necessary to design a robust gain-scheduled controller. The next section presents the results of the proposed robust controller and a comparison with a fixed-gain controller based on the nominal model with average compositions at one set-point.

\section{Control design}

1) Fixed-gain controller design: The first approach is to design a discrete MIMO PI controller $C_{n}(z)$ using only the nominal model $G_{n}$ which is computed using the average compositions of the input materials given in Table I at the setpoints $\alpha_{c}=100$ and $\gamma_{c}=1.3$. Since the transfer functions $g_{i j}(s)$ are equal they can be replaced by one single transfer function

$$
g(s)=\frac{e^{-T_{d} s}}{\left(T_{i} s+1\right)\left(T_{f} s+1\right)}
$$

where $T_{i}=2 \mathrm{~min}$ is the time constant of the dynamics of the feeders, $T_{f}=4.64 \mathrm{~min}$ is the time constant of the noise filter and $T_{d}=2.5 \mathrm{~min}$ is the delay accounting for the transport and measurement delays.

The gains of the nominal model under the given conditions are shown in Table II. The controller is a $2 \times 2$ matrix where all basis functions $\phi_{p q}$ are given by

$$
\phi_{p q}=\left[\frac{z}{z-1}, \frac{1}{z-1}\right]^{T}
$$


TABLE I

Average weight percentage oxide compositions of the 3 input materials, corresponding to $W^{T}$.

\begin{tabular}{|c|c|c|c|c|}
\hline Material & $\mathrm{CaO}$ & $\mathrm{SiO}_{2}$ & $\mathrm{Al}_{2} \mathrm{O}_{3}$ & $\mathrm{Fe}_{2} \mathrm{O}_{3}$ \\
\hline Limestone & 51.9280 & 3.5851 & 1.2953 & 0.5773 \\
\hline Marl & 10.3746 & 53.7396 & 13.1175 & 4.9608 \\
\hline Iron Correctives & 1.2102 & 25.4592 & 3.3082 & 64.0195 \\
\hline
\end{tabular}

TABLE II

Static gains of the nominal model

\begin{tabular}{|c|c|c|c|}
\hline$k_{11}$ & $k_{12}$ & $k_{21}$ & $k_{22}$ \\
\hline $1.55 \times 10^{4}$ & $-4.32 \times 10^{3}$ & 80.46 & 86.58 \\
\hline
\end{tabular}

Defining the desired open loop transfer function

$$
L_{D}=\left[\begin{array}{cc}
\frac{\omega_{c}}{z-1} & 0 \\
0 & \frac{\omega_{c}}{z-1}
\end{array}\right]
$$

where $\omega_{c}=0.4489$ and solving the optimization problem stated by Eq. (8) with $m=1$ and $N=50$ equally spaced frequency points between $\frac{\omega_{c}}{N}$ and $\frac{\pi}{T_{s}}$, the following controller is obtained:

$$
C_{n}(z)=\left[\begin{array}{cc}
\frac{10^{-4}\left(1.1-z^{-1}\right)}{1-z^{-1}} & \frac{0.006-0.005 z^{-1}}{1-z^{-1}} \\
\frac{10^{-4}\left(-1.03+009 z^{-1}\right)}{1-z^{-1}} & \frac{0.0200-0.0180 z^{-1}}{1-z^{-1}}
\end{array}\right]
$$

2) Robust gain-scheduled controller design: Here, a robust gain-scheduled will be designed using the LPV models $G_{i}(\theta)$, where $i=1, \ldots, 8$, computed in Section IV-B. Consider the gain-scheduled controller $C_{r}(z, \theta)$

$$
C_{r}(z, \theta)=\left[\begin{array}{ll}
\frac{\rho_{111}(\theta)+\rho_{112}(\theta) z^{-1}}{1-z^{-1}} & \frac{\rho_{121}(\theta)+\rho_{122}(\theta) z^{-1}}{1-z^{-1}} \\
\frac{\rho_{211}(\theta)+\rho_{211}(\theta) z^{-1}}{1-z^{-1}} & \frac{\rho_{221}(\theta)+\rho_{222}(\theta) z^{-1}}{1-z^{-1}}
\end{array}\right]
$$

Every element $K_{p q}(z, \theta)$ of the multivariable controller is a discrete-time PI whose basis functions are given by Eq. (24) and whose gains are linearly parametrized as shown below.

$$
\begin{gathered}
\rho_{p q 1}=\left(\nu_{p q 1,1}\right)^{T} \cdot\left[\begin{array}{l}
\alpha_{c} \\
\gamma_{c}
\end{array}\right]+\nu_{p q 1,0} \\
\rho_{p q 2}=\left(\nu_{p q 2,1}\right)^{T}\left[\begin{array}{l}
\alpha_{c} \\
\gamma_{c}
\end{array}\right]+\nu_{p q 2,0}
\end{gathered}
$$

where $\nu_{p q i, 1}$ is a vector of length 2 and $\nu_{p q i, 0}$ is a scalar. The set-points $\alpha_{c}$ and $\gamma_{c}$ can vary within the ranges $[96,103]$ and $[1.3,2.1]$, respectively.

Define $u$ and $v$ the coordinates used to grid the allowable ranges of $\alpha_{c}$ and $\gamma_{c}$. The open loop transfer matrix is then $L_{i}(\theta(u, v))=G_{i}(\theta(u, v)) C_{r}(\theta(u, v))$. Thus, the robust gainscheduled controller is given by the following optimization problem:

$$
\min _{\rho} \sum_{u=1}^{u_{0}} \sum_{v=1}^{v_{0}} \sum_{i=1}^{m} \sum_{k=1}^{N}\left\|L_{i}\left(j \omega_{k}, \theta(u, v)\right)-L_{D i}\left(j \omega_{k}\right)\right\|_{F}
$$

subject to

$$
\begin{array}{r}
r_{q i}\left(\omega_{k}\right)-\frac{R_{e}\left\{\left[1+L_{D i q}\left(-j \omega_{k}\right)\right]\left[1+L_{i q q}\left(j \omega_{k}, \theta(u, v)\right)\right]\right\}}{\left|\left[1+L_{D i q}\left(j \omega_{k}\right)\right]\right|}<0 \\
q=1, \ldots, n_{0} \quad k=1, \ldots, N \quad i=1, \ldots, m \quad \text { (30) }
\end{array}
$$

The set-point ranges are equally divided into $u_{0}=5$ and $v_{0}=5$ points. The number of LPV models is $m=8$ and the frequency range is gridded by $N=50$ linearly spaced points. Both controllers are compared in two different cases. Firstly, only the nominal model is used and a set-point tracking is performed. Next, the controllers is evaluated using another model of the set.

\section{Controller evaluation}

1) Nominal model: In this simulated example, $\gamma_{c}$ changes within 1.3 and 2.1. Gaussian measurement noise is added to $x$ with zero-mean and variance $\mu=[0.12,0.04,0.06,0.003]^{T}$, which are typical values in the industry. Figures 2 and 3 compare the outputs and inputs for the nominal controller with fixed gains with those of the gain-scheduled controller. The performance of the fixed-gain controller deteriorates after the set-point change since the system gains increase. The decoupling of the gain-scheduled controller is also better as it can be seen in Fig. 2. Table III compares the performance of both controllers. The integral absolute error (IAE) and the integral square error (ISE) of the outputs $\alpha$ and $\gamma$ for the gain-scheduled controller are up to $83 \%$ and $42.86 \%$ lower, respectively.

It is worth to point out that for both controllers, the controlled variables are the transformed outputs $y_{1}$ and $y_{2}$ which automatically guarantees the tracking of the quality modules $\alpha$ and $\gamma$.

TABLE III

Comparison of the performance of the fixed-gain controller (PI) and the gain-scheduled controller (GS).

\begin{tabular}{|c|c|c|c|c|}
\hline & $\alpha_{G S}$ & $\alpha_{P I}$ & $\gamma_{G S}$ & $\gamma_{P I}$ \\
\hline IAE & $2.5 \times 10^{3}$ & $2.9 \times 10^{3}$ & 417 & 765.1 \\
\hline ISE & $1.4 \times 10^{3}$ & $2 \times 10^{3}$ & 160.8 & 194.7 \\
\hline
\end{tabular}

2) Uncertain model: Here, the performance of each controller is evaluated for rejecting disturbances in the compositions of the input materials. In order to evaluate the controllers, the following simulation is carried out. Starting from the nominal average composition matrix $W_{0}$ (see Table I) a disturbance variation $\Delta W_{d}$ is added such that the new composition matrix becomes $W_{d}=W_{0}+\Delta W_{d}$ at time 50 min. After stabilization, a set-point change is performed. The variation is computed using the bounds of $\Delta w_{e}$ obtained using PCA as presented above. Matrix $\Delta W_{d}$ is generated using $\Delta w_{1}=\Delta w_{1}^{-}, \Delta w_{2}=\Delta w_{2}^{+}$and $\Delta w_{3}=\Delta w_{3}^{+}$and is given by

$$
\Delta W_{d}=\left[\begin{array}{ccc}
1.5845 & -2.6875 & -0.0722 \\
-1.8836 & 5.3333 & 2.5874 \\
-0.6144 & 0.1912 & 0.7530 \\
-0.2852 & -0.3930 & -1.7007
\end{array}\right]
$$



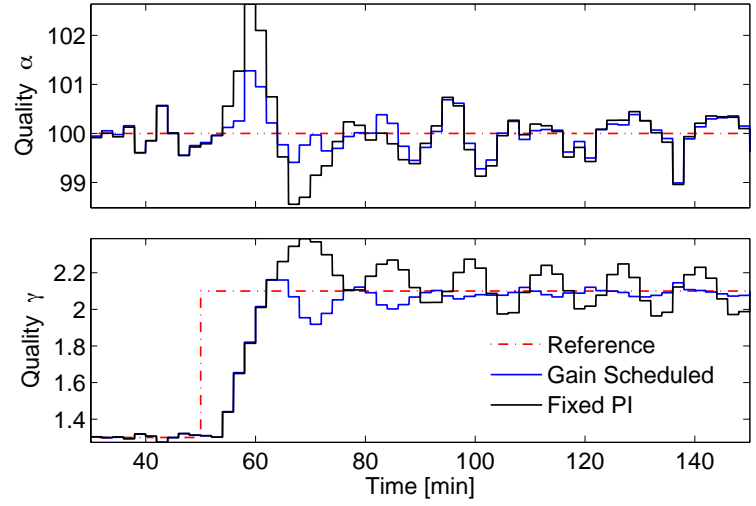

Fig. 2. Quality modules setpoint tracking: nominal model
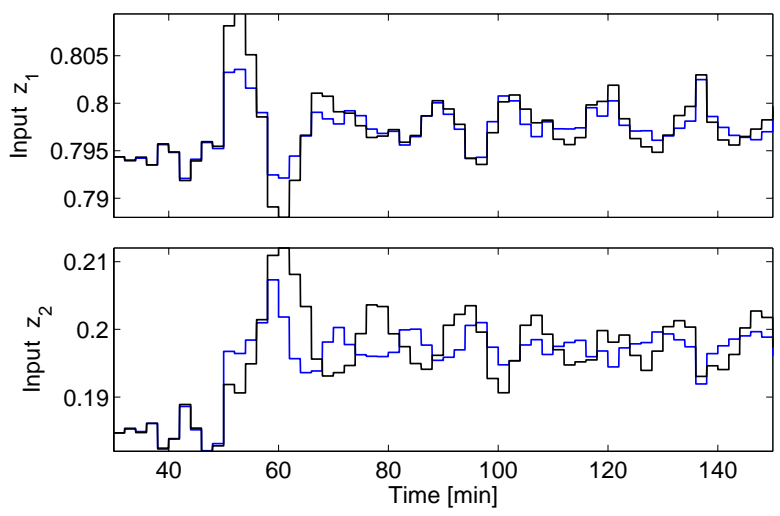

Fig. 3. Feeders proportions setpoint tracking: nominal model

As it can be seen in Fig. 4 both controllers are able to reject the disturbances and reach to the steady state after 50 minutes. Nevertheless, it can be seen that the fixedgain controller is much more oscillatory after the inlet composition change and the set-point change, while the robust gain-scheduled controller keeps its performance close to the nominal despite of the increased system gains after disturbance.

\section{CONCLUSIONS}

This article presented a methodology to design robust gain-scheduled MIMO controller with application to a blending control problem of the raw mix quality in the cement industries. The robust gain-scheduled controller, which is obtained from a one-step convex optimization problem, showed considerable advantage when compared to a fixedgain controller in the case study example.

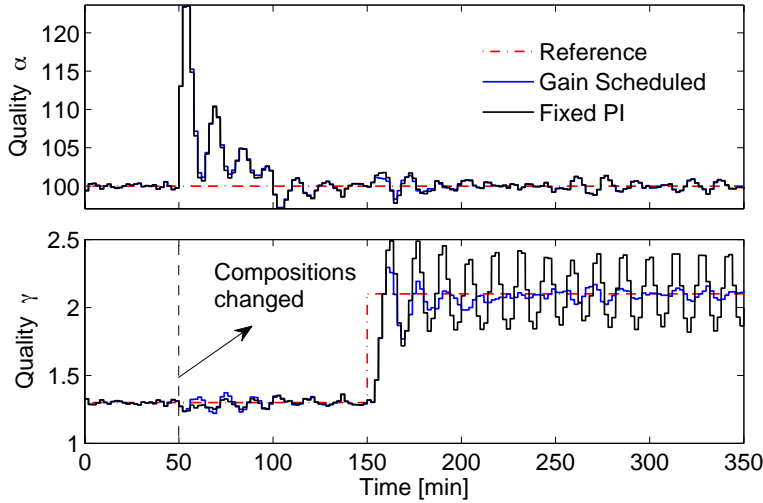

Fig. 4. Quality modules: uncertain model

Moreover, by using PCA it has been possible to describe the uncertainties of the composition of the input materials in a way that the conservatism is reduced as well as the number of models necessary to design the controller.

\section{ACKNOWLEDGMENTS}

Lolla Tapovan is acknowledged for his preliminary work on this project during an internship in Automatic Control Laboratory of EPFL.

\section{REFERENCES}

[1] A. Kural and C. Oezsoy, "Identification and control of the raw material blending process in cement industry," International Journal of Adaptive Control and Signal Processing, vol. 18, no. 5, pp. 427-442, 2004.

[2] C. Banyasz, L. Keviczky, and I. Vajk, "A novel adaptive control system for raw material blending," IEEE Control Systems Magazine, vol. 23, no. 1, pp. 87-96, 2003.

[3] A. K. Swain, "Material mix control in cement plant automation," IEEE Control Systems Magazine, vol. 15, no. 4, pp. 23-27, 1995.

[4] C. Ozsoy, A. Kural, M. Cetinkaya, and S. Ertug, "Constrained mimo self-tuning composition control in cement industry," in Proceedings of 7 th IEEE International Conference on Emerging Technologies and Factory Automation, vol. 2, 1999, pp. 1021-1028.

[5] P. P. Bonissone and Y. T. Chen, "System and method for providing raw mix proportioning control in a cement plant with a gradient-based predictive controller," 2000, US Patent 6,120,173.

[6] M. A. Eggimann, "Réglage sous contraintes de saturation par programmation lineaire," Ph.D. dissertation, Ecole Polytechnique Fédérale de Lausanne, Laboratoire d'Automatique, 1015 Lausanne, Switzerland, 1990.

[7] G. Galdos, A. Karimi, and R. Longchamp, " $h_{\infty}$ controller design for spectral MIMO models by convex optimization," Journal of Process Control, vol. 20, no. 10, pp. 1175 - 1182, 2010.

[8] A. Karimi and G. Galdos, "Fixed-order $H_{\infty}$ controller design for nonparametric models by convex optimization," Automatica, vol. 46, no. 8, pp. 1388-1394, 2010.

[9] H. H. Rosenbrock, State-space and multivariable theory. London Nelson, 1970

[10] M. Kunze, A. Karimi, and R. Longchamp, "Gain-scheduled controller design by linear programming," in European Control Conference, July 2007, pp. 5432-5438. 